\title{
Long range rapidity correlations between the transverse momentum and the multiplicities in light-nuclei collisions
}

\author{
Evgeny Andronov ${ }^{* \dagger}$ \\ Saint Petersburg State University \\ E-mail: evgeny.andronov1@gmail.com
}

Vladimir Vechernin

Saint Petersburg State University

E-mail: vechernin@gmail.com

\begin{abstract}
During recent decades the long-range rapidity correlations phenomenon was studied both experimentally and theoretically in relativistic nuclear physics. The correlations between the multiplicities (n-n) and the transverse momentum and the multiplicity (pT-n) of charge particles are analyzed in the framework of the simple string inspired model with two types of sources. The sources of the first type (primary emitters) correspond to the initial strings formed in a hadronic collision. We introduce the sources of the second type (secondary emitters), which imitate the appearance of the emitters of a new kind resulting from interaction (fusion) of the initial strings. The model enabled to describe effectively the influence of the string fusion effects on the strength both the n-n and the pT-n correlations.

It was found that in the region, where the secondary emitters start to produce, the calculation results predict the non-monotonic behaviour of the $n-n$ and pT-n correlation coefficients with the growth of the mean number of initial strings, i.e. with the increase of the collision centrality. We show also that the increase of the event-by-event fluctuation in the number of primary strings leads to the change of the pT-n correlation sign from negative to positive. Similar behaviour of the pT- $n$ correlation coefficient was observed experimentally in the lead-lead collisions at the top SPS energy. One can try to search all these signatures of string collective phenomena in interactions of various nuclei at different energies varying the class of collision centrality and its width.
\end{abstract}

XXII International Baldin Seminar on High Energy Physics Problems,

15-20 September 2014

JINR, Dubna, Russia

\footnotetext{
*Speaker.

${ }^{\dagger}$ The work was supported by the SPbSU research grant 11.38.197.2014.

$¥$ The work was supported by the RFFI grant 15-02-02097 and the SPbSU research grant 11.38.197.2014.
} 


\section{Introduction}

This work is devoted to the analysis of long-range rapidity correlations (or forward-backward correlations), being a unique tool sensitive to the collective effects in high energy ion collisions. Since processes of the soft multi-particle production could not be described within a perturbative approach of the fundamental theory of strong interactions - QCD, one has to apply phenomenological models, such as models with a two-stage scenario of particle production [1, 2, 3, 4]. In this scheme at the first stage several extended in rapidity color strings are stretched between colliding ions. Then, at the second stage, they decay into observed charged particles, leading to the presence of the long-range rapidity correlations between multiplicities. The string concept is widely used in the scope of the modern Monte Carlo event generators, such as DPM [2], QGSM [4], VENUS [5], EPOS [6].

Number of quark-gluon strings would increase with the growing collision energy and atomic number of participants, which could result in the interaction between strings (fusion) due to the finite transverse size of a string and the limited transverse overlapping area in a collision [7, 8]. This fusion is connected with the interaction of the color fields inside strings, which, in turn, change resulting multiplicity and transverse momentum distributions. Therefore, it was suggested to consider several types of correlations $[9,10]$ to study this collective phenomena: the correlation between multiplicities of charged particles in two rapidity windows separated by some gap and the correlation between event-mean value of transverse momentum and multiplicity in these windows. The strength of the correlations is characterized by the correlation parameters:

$$
b_{n-n}^{a b s}=\frac{\left\langle n_{F} n_{B}\right\rangle-\left\langle n_{F}\right\rangle\left\langle n_{B}\right\rangle}{\left\langle n_{F}^{2}\right\rangle-\left\langle n_{F}\right\rangle^{2}} ; \quad b_{p_{t}-n}^{a b s}=\frac{\left\langle n_{F} p_{t B}\right\rangle-\left\langle n_{F}\right\rangle\left\langle p_{t B}\right\rangle}{\left\langle n_{F}^{2}\right\rangle-\left\langle n_{F}\right\rangle^{2}}
$$

The averaging $\langle\cdots\rangle$ in Eqs. (1.1) is over all events. The values, defined in Eqs. (1.1), depend on the widths of the forward and backward windows. Therefore, usually [11], normalized versions of the correlation parameters are considered, where following replacements $n \rightarrow \frac{n}{\langle n\rangle}, p_{t} \rightarrow \frac{p_{t}}{\left\langle p_{t}\right\rangle}$ take place:

$$
b_{n-n}^{r e l}=\frac{\left\langle n_{F}\right\rangle}{\left\langle n_{B}\right\rangle} b_{n-n}^{a b s} ; \quad b_{p_{t}-n}^{r e l}=\frac{\left\langle n_{F}\right\rangle}{\left\langle p_{t B}\right\rangle} b_{p_{t}-n}^{a b s}
$$

Nevertheless, these substitutions enable us to reduce a trivial dependence of the correlation parameters only on the width of the backward rapidity window.

In this paper we study $n-n$ and $p T-n$ correlations in the framework of the model with two types of strings [12], which effectively reproduces string fusion effects. This approach is the simplest possible modification of the model with identical emitters [13], that can be applied in the case of $p p$ collisions where string fusion effects are negligible in the first approximation.

\section{Model with strings of two types}

\subsection{General formalism}

In the present model, it is proposed to consider quark-gluon strings of two types. The strings of the first type correspond to the initial strings formed in a hadronic collision. The strings of the 
second type imitate the appearance of the emitters of a new kind resulting from fusion of the initial strings. We suppose that in a single event $N_{1}$ strings of the first type and $N_{2}$ strings of the second type are created with probability $q\left(N_{1}, N_{2}\right)$ :

$$
\sum_{N_{1}, N_{2}} q\left(N_{1}, N_{2}\right)=1
$$

We introduce following notations for the first and second moments of this joint distribution :

$$
\begin{aligned}
& \sum_{N_{1}, N_{2}} q\left(N_{1}, N_{2}\right) N_{1}=\overline{N_{1}} ; \sum_{N_{1}, N_{2}} q\left(N_{1}, N_{2}\right) N_{2}=\overline{N_{2}} ; \sum_{N_{1}, N_{2}} q\left(N_{1}, N_{2}\right) N_{1} N_{2}=\overline{N_{1} N_{2}} ; \\
& \sum_{N_{1}, N_{2}} q\left(N_{1}, N_{2}\right) N_{1}^{2}=\overline{N_{1}^{2}} ; \sum_{N_{1}, N_{2}} q\left(N_{1}, N_{2}\right) N_{2}^{2}=\overline{N_{2}^{2}} ; \\
& D_{N_{1}}=\overline{N_{1}^{2}}-{\overline{N_{1}}}^{2} ; D_{N_{2}}=\overline{N_{2}^{2}}-{\overline{N_{2}}}^{2} ; \operatorname{cov}\left(N_{1}, N_{2}\right)=\overline{N_{1} N_{2}}-\overline{N_{1}} \cdot \overline{N_{2}}
\end{aligned}
$$

In order to characterize string fragmentation we put into consideration probabilities to get a particular number of charged particles in two windows from a string of the first and second types: $p^{(1)}\left(F_{i}^{(1)}, B_{i}^{(1)}\right), p^{(2)}\left(F_{j}^{(2)}, B_{j}^{(2)}\right)$. Here, indices (1), (2) represent the type of string, and latin indices stand for the string number.

Since we consider the long-range correlations, a gap between the forward and backward windows could be chosen sufficiently large to consider numbers of particles produced in these windows from one string as uncorrelated:

$$
p^{(1)}\left(F_{i}^{(1)}, B_{i}^{(1)}\right)=p_{F^{(1)}}\left(F_{i}^{(1)}\right) \cdot p_{B^{(1)}}\left(B_{i}^{(1)}\right) ; \quad p^{(2)}\left(F_{j}^{(2)}, B_{j}^{(2)}\right)=p_{F^{(2)}}\left(F_{j}^{(2)}\right) \cdot p_{B^{(2)}}\left(B_{j}^{(2)}\right)
$$

The first and seconds moments of the new single-string distributions are denoted as follows:

$$
\begin{aligned}
& \sum_{F_{i}^{(1)}} p_{F^{(1)}}\left(F_{i}^{(1)}\right) F_{i}^{(1)}=\bar{\mu}_{F^{(1)}} ; \sum_{F_{j}^{(2)}} p_{F^{(2)}}\left(F_{j}^{(2)}\right) F_{j}^{(2)}=\bar{\mu}_{F^{(2)}} ; \\
& \sum_{B_{i}^{(1)}} p_{B^{(1)}}\left(B_{i}^{(1)}\right) B_{i}^{(1)}=\bar{\mu}_{B^{(1)}} ; \sum_{B_{j}^{(2)}} p_{B^{(2)}}\left(B_{j}^{(2)}\right) B_{j}^{(2)}=\bar{\mu}_{B^{(2)}} ; \\
& D_{\mu_{F^{(1)}}}=\overline{\mu_{F^{(1)}}^{2}}-\bar{\mu}_{F^{(1)}}^{2} ; D_{\mu_{F^{(2)}}}=\overline{\mu_{F^{(2)}}^{2}}-\bar{\mu}_{F^{(2)}}^{2} \\
& D_{\mu_{B^{(1)}}}=\overline{\mu_{B^{(1)}}^{2}}-\bar{\mu}_{B^{(1)}}^{2} ; D_{\mu_{B^{(2)}}}=\overline{\mu_{B^{(2)}}^{2}}-\bar{\mu}_{B^{(2)}}^{2} .
\end{aligned}
$$

Assumption (2.3) can be tested in experiments where fixed number of quark-gluon strings is created. In this model long-range correlations originate only from the fluctuations in number and type of strings, what will lead to the absence of long-range correlations in the mentioned experiments without these fluctuations. Electron-positron collisions at low energies could be considered as such an experiment due to formation of a single $q-\bar{q}$ string. In [14] it was found that for $e^{+} e^{-}$collisions at $\sqrt{s}=29 \mathrm{GeV}$ the $n-n$ correlation strength for two symmetrical windows with large gap $\Delta y=2$ is negligible: $b_{n-n}^{a b s}=0.002 \pm 0.006$. Similar effect can be seen in low-energy proton-proton collisions with two quark-diquark strings. The $n-n$ correlation strength for $p p$ collisions at $\sqrt{s}=24 \mathrm{GeV}$ [15] for two symmetrical windows with $\Delta y=2$ is also close to zero: $b_{n-n}^{a b s}=0.032 \pm 0.015$. 
It was shown [12] that within the proposed model one can get following contributions to the $n-n$ correlation parameter, using notations from Eqs. (2.2), (2.4):

$$
\begin{aligned}
& \left\langle n_{F}{ }^{2}\right\rangle=\overline{N_{1}{ }^{2}} \bar{\mu}_{F^{(1)}}^{2}+\overline{N_{2}{ }^{2}} \bar{\mu}_{F^{(2)}}^{2}+2 \overline{N_{1} N_{2}} \bar{\mu}_{F^{(1)}} \bar{\mu}_{F^{(2)}}+\overline{N_{1}} D_{\mu_{F^{(1)}}}+\overline{N_{2}} D_{\mu_{F^{(2)}}} \\
& \left\langle n_{F}\right\rangle=\bar{N}_{1} \bar{\mu}_{F^{(1)}}+\overline{N_{2}} \bar{\mu}_{F^{(2)}} \\
& \left\langle n_{B}\right\rangle=\overline{N_{1}} \bar{\mu}_{B^{(1)}}+\overline{N_{2}} \bar{\mu}_{B^{(2)}} \overline{N_{2}{ }^{2}} \bar{\mu}_{B^{(2)}} \bar{\mu}_{F^{(2)}}+\overline{N_{1} N_{2}}\left(\bar{\mu}_{B^{(1)}} \bar{\mu}_{F^{(2)}}+\bar{\mu}_{B^{(2)}} \bar{\mu}_{F^{(1)}}\right) \\
& \left\langle n_{B} n_{F}\right\rangle={\overline{N_{1}}}^{2} \bar{\mu}_{B^{(1)}} \bar{\mu}_{F^{(1)}}+
\end{aligned}
$$

Additional contributions to the $p T-n$ correlation coefficient are listed below:

$$
\begin{gathered}
\left\langle p_{t B}\right\rangle=\sum_{B^{(1)}, B^{(2)}} \frac{\overline{k_{1}} B^{(1)}+\overline{k_{2}} B^{(2)}}{B^{(1)}+B^{(2)}} \sum_{N_{1}, N_{2}} q\left(N_{1}, N_{2}\right) P_{N_{1}}\left(B^{(1)}\right) P_{N_{2}}\left(B^{(2)}\right) \\
\left\langle p_{t B} n_{F}\right\rangle=\sum_{B^{(1)}, B^{(2)}} \frac{\overline{k_{1}} B^{(1)}+\overline{k_{2}} B^{(2)}}{B^{(1)}+B^{(2)}} \sum_{N_{1}, N_{2}} q\left(N_{1}, N_{2}\right)\left(N_{1} \bar{\mu}_{F^{(1)}}+N_{2} \bar{\mu}_{F^{(2)}}\right) P_{N_{1}}\left(B^{(1)}\right) P_{N_{2}}\left(B^{(2)}\right)
\end{gathered}
$$

where $\overline{k_{1}}$ and $\overline{k_{2}}$ are the mean transverse momenta of charged particles in the backward window from one string of the first and second types, $B^{(1)}$ and $B^{(1)}$ are the total multiplicities in the backward window from all strings of the first/second type and following probability distributions were introduced:

$$
\begin{aligned}
& P_{N_{1}}\left(B^{(1)}\right)=\sum_{\left\{B_{i}^{(1)}\right\}} \delta_{B^{(1)}, \Sigma_{i=1}^{N_{1}} B_{i}^{(1)}} \prod_{i=1}^{N_{1}} p_{B^{(1)}}\left(B_{i}^{(1)}\right) \\
& P_{N_{2}}\left(B^{(2)}\right)=\sum_{\left\{B_{j}^{(2)}\right\}} \delta_{B^{(2)}, \Sigma_{j=1}^{N_{2}} B_{j}^{(2)}} \prod_{j=1}^{N_{2}} p_{B^{(2)}}\left(B_{j}^{(2)}\right)
\end{aligned}
$$

Substituting Eqs. (2.5)-(2.10) in Eqs. (1.1) gives the following expression for the n-n correlation parameter:

$$
b_{n-n}^{a b s}=\frac{D_{N_{1}} \bar{\mu}_{F^{(1)}} \bar{\mu}_{B^{(1)}}+D_{N_{2}} \bar{\mu}_{F^{(2)}} \bar{\mu}_{B^{(2)}}+\operatorname{cov}\left(N_{1}, N_{2}\right)\left(\bar{\mu}_{F^{(1)}} \bar{\mu}_{B^{(2)}}+\bar{\mu}_{F^{(2)}} \bar{\mu}_{B^{(1)}}\right)}{D_{N_{1}} \bar{\mu}_{F^{(1)}} 2+D_{N_{2}} \bar{\mu}_{F^{(2)}}{ }^{2}+2 \operatorname{cov}\left(N_{1}, N_{2}\right) \bar{\mu}_{F^{(1)}} \bar{\mu}_{F^{(2)}}+\bar{N}_{1} D_{\mu_{F^{(1)}}}+\overline{N_{2}} D_{\mu_{F^{(2)}}}}
$$

and for the pT-n correlation parameter:

$$
b_{p_{t}-n}^{a b s}=\frac{\left\langle n_{F} p_{t B}\right\rangle-\left(\overline{N_{1}} \bar{\mu}_{F^{(1)}}+\bar{N}_{2} \bar{\mu}_{F^{(2)}}\right)\left\langle p_{t B}\right\rangle}{D_{N_{1}} \bar{\mu}_{F^{(1)}}{ }^{2}+D_{N_{2}} \bar{\mu}_{F^{(2)}}{ }^{2}+2 \operatorname{cov}\left(N_{1}, N_{2}\right) \bar{\mu}_{F^{(1)}} \bar{\mu}_{F^{(2)}}+\overline{N_{1}} D_{\mu_{F^{(1)}}}+\overline{N_{2}} D_{\mu_{F^{(2)}}}}
$$

If properties of both types of strings are identical and $N_{1}+N_{2}=N$, then we get:

$$
\begin{aligned}
& b_{n-n}^{a b s}=\frac{D_{N} \bar{\mu}_{F} \bar{\mu}_{B}}{D_{N} \bar{\mu}_{F}^{2}+\bar{N} D_{\mu_{F}}} \\
& b_{p_{t}-n}^{a b s}=0
\end{aligned}
$$

which coincides with the previously obtained results in the model with independent emitters of one type [13].

Note that we do not need to know exact form of the single-particle momentum distributions to calculate the $p T-n$ correlation coefficient. The expression in Eq. (2.13) is governed only by mean values $\overline{k_{1}}$ and $\overline{k_{2}}$ and multiplicity distributions. 


\subsection{Mechanism of strings formation}

Following the Regge-Gribov theory [16, 17], we suppose that multi-pomeron exchange processes, taking place in high energy hadron-hadron or nucleus-nucleus collisions, correspond to creation of $q-q q$ and $q-\bar{q}$ strings, with two strings originating from each cut-pomeron. Note that strings could be stretched between valence quarks and valence diquarks, valence quarks and sea antiquarks, valence diquarks and sea quarks, sea quarks and sea antiquarks. In this model, we assume that all these color emitters contribute to both considered rapidity windows (they are infinite in this sense) and have the same fragmentation properties. Thus, $N=2 H$ identical strings are created in an event with $H$ cut pomerons. Next, one can assemble $N$ emitters into $\frac{N}{2}$ pairs and introduce probability $r$ of pair transformation into a new fused string. Therefore, $N_{2}$ new strings will be created and $N_{1}=N-2 N_{2}$ primary strings will left from the initial configuration. Note that the concept of pair string fusion evidently can not be applied for the collisions of heavy ions, because in this case a lot of strings overlap in the transverse plane. As a result, for light ions, we will get a binomial distribution for the number of the new emitters with the fixed $N: P_{N}\left(N_{2}\right)=C_{\frac{N}{2}}^{N_{2}} \cdot r^{N_{2}} \cdot(1-r)^{\frac{N}{2}-N_{2}}$ and

$$
\sum_{N_{1}, N_{2}} q\left(N_{1}, N_{2}\right) \cdots=\sum_{N=0}^{\infty} \sum_{N_{2}=0}^{\frac{N}{2}} q(N) \cdot P_{N}\left(N_{2}\right) \cdots=\sum_{H=0}^{\infty} \sum_{N_{2}=0}^{H} q(H) \cdot P_{H}\left(N_{2}\right) \cdots
$$

where $q(N)$ (or $q(H)$ ) is the probability of $N$ primary strings (or $H$ cut-pomerons) formation. Since we consider events with at least one cut-pomeron, the first summation in the right-hand side of Eq. (2.16) should start from unity. In order to keep proper normalization $q(H)$ is substituted by $\tilde{q}(H)$ :

$$
\tilde{q}(H)=\frac{q(H)}{1-q(0)}
$$

Evidently, this modification will change the average number of primary string in a single event $\bar{N}$ in the same way:

$$
\tilde{N}=\frac{\bar{N}}{1-q(0)}
$$

Note that the fluctuation in number of strings in pp and AA collisions is not poissonian and hence the scaled variance $\omega_{N} \equiv \frac{D_{N}}{\tilde{N}} \neq 1$. Its value, as well as $\tilde{N}$, depends on the collision energy and centrality.

As a next step, we make an assumption about connection between fragmentation properties of strings of two types. It is usually supposed that when two strings fuse, the colour charge modifies $[10,18,19]$

$$
Q_{2}=\sqrt{2} Q
$$

where $Q$ and $Q_{2}$ are the colour charges inside primary and new emitters. This leads to the following relations:

$$
\bar{\mu}_{F^{(2)}}=\sqrt{2} \bar{\mu}_{F^{(1)}} ; \quad \bar{\mu}_{B^{(2)}}=\sqrt{2} \bar{\mu}_{B^{(1)}} ; \quad \overline{k_{2}}=2^{\frac{1}{4}} \overline{k_{1}}
$$

In principle, the right-hand sides in (2.19)-(2.20) should me multiplied by a factor proportional to the area of the overlap, but we suppose that the process of fusion create strings of new type as a result of complete overlapping of two primary strings. 
In order to keep the scaled variances independent on the string type $\frac{D_{F^{(2)}}}{\bar{\mu}_{F^{(2)}}}=\frac{D_{\mu_{F^{(1)}}}}{\overline{\mu_{F(1)}}} \equiv \omega\left[\mu_{F^{(1)}}\right]$, $\frac{D_{B^{(2)}}}{\bar{\mu}_{B^{(2)}}}=\frac{D_{B_{B^{(1)}}}}{\bar{\mu}_{B^{(1)}}} \equiv \omega\left[\mu_{B^{(1)}}\right]$ we choose

$$
D_{F^{(2)}}=\sqrt{2} D_{\mu_{F^{(1)}}} ; \quad D_{\mu_{B^{(2)}}}=\sqrt{2} D_{\mu_{B^{(1)}}}
$$

This assumption is based on the idea that both primary and new strings are homogeneously extended in rapidity and consequently have a Poisson distribution in the number of produced particles in the backward and forward windows, which leads to:

$$
\omega\left[\mu_{F^{(1)}}\right]=\omega\left[\mu_{B^{(1)}}\right]=1
$$

In our previous study [12] it was shown that in this approach the $p T-n$ correlation coefficient can reach only non-positive values, with zero correlations obtained for $r=0$ and $r=1$ parameters ${ }^{1}$. Note that experimentally both negative and positive $p T-n$ correlations were observed [21].

Incompleteness of description in [12] comes from the independence of fusion parameter $r$ on the number of initially created quark-gluon strings $N$. It is natural to expect that with growing string density the probability of emitter interaction should also rise. We use the following parametrization of $r(N)$ :

$$
r(N)=\frac{1}{1+e^{-\frac{N-N_{0}}{\delta N}}}
$$

where $N_{0}$ and $\delta N$ are two additional parameters. This function tends to zero at small $N$, representing the absence of fusion, and to unity at high $N$, representing complete fusion.

Substituting function (2.23) into formulae (2.16) leads to an impossibility of analytical evaluation of the average values (2.5)-(2.10) included in the definitions of the $n-n$ and $p T-n$ correlation coefficients. In order to perform these calculations numerically a toy Monte-Carlo model was developed. An event generation in a code consists in string configuration creation (generation of $N$ and $N_{2}$ in accordance with $q(N)$ and $P_{N}\left(N_{2}\right)$ ), followed by the computation of multiplicities in two windows from this configuration (generation of $n_{F}$ and $n_{B}$ ). By repeating this procedure one can get approximate results for (2.5)-(2.10) and, consequently, for the correlation parameter (1.2).

\subsection{Results}

\subsubsection{Test of Monte-Carlo code}

Calculations were performed for different probability distributions $q(H)$. It turned out that for the configurations with the fixed number of cut-pomerons (or primary strings $\omega[N]=\frac{D_{N}}{\bar{N}}=0$ ) one can get an analytical result for the $n-n$ correlation coefficient:

$$
\begin{aligned}
b_{n-n}^{a b s} & =\frac{\bar{\mu}_{B} \cdot \bar{\mu}_{F} \cdot N \cdot r(N) \cdot(1-r(N)) \cdot(3-2 \sqrt{2})}{\bar{\mu}_{F}^{2} \cdot N \cdot r(N) \cdot(1-r(N)) \cdot(3-2 \sqrt{2})+\bar{\mu}_{F} \cdot N \cdot\left(1-r(N)+\frac{\sqrt{2}}{2} r(N)\right)} \\
b_{n-n}^{r e l} & =b_{n-n}^{a b s} \cdot \frac{\bar{\mu}_{F}}{\bar{\mu}_{B}}
\end{aligned}
$$

\footnotetext{
${ }^{1}$ In this two extreme cases there are no fluctuations in types of strings.
} 

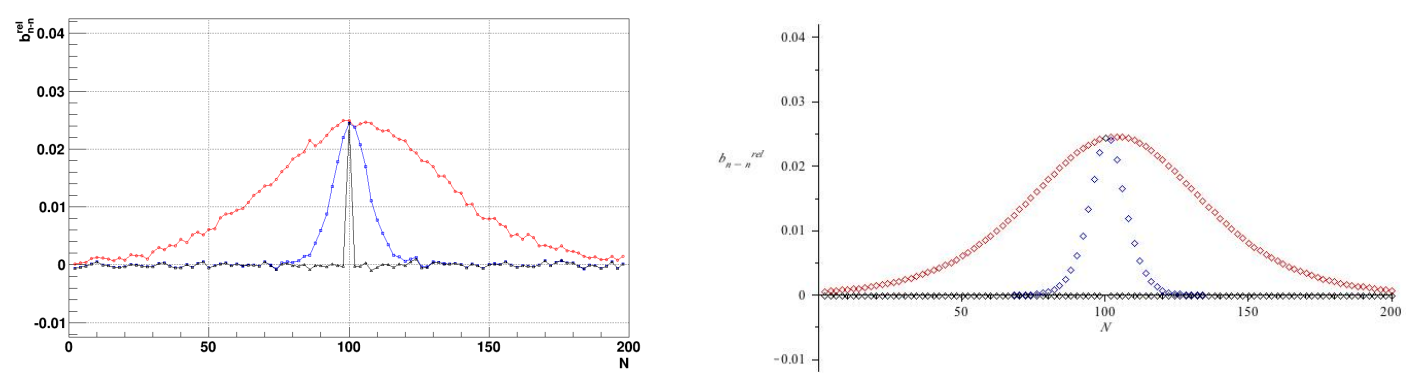

Figure 1: The $n-n$ correlation coefficient in the relative variables for $N_{0}=100, \bar{\mu}_{F}=\bar{\mu}_{B}=0.5$ as a function of the fixed number of primary strings $N$ : Monte-Carlo calculations (left), analytic calculations (right). Red lines correspond to $\delta N=20$, blue $-\delta N=4$ and black $-\delta N=0.2$.

Thus, our Monte-Carlo code could be tested by making comparison between its results and expression in Eq. (2.25). This comparison is shown in Fig. 1 for $N_{0}=100, \bar{\mu}_{F}=\bar{\mu}_{B}=0.5$ and several $\delta N$ magnitudes. Monte-Carlo simulations (Fig. 1 (left)) show a perfect agreement with the analytical calculations (Fig. 1 (right)). Moreover, we can observe an absence of correlations at small and high values of $N$, corresponding to $r \approx 0$ and $r \approx 1$, for different values of parameter $\delta N$, which is due to the negligible fluctuations in type of strings and zero fluctuations in number of strings.

Note that for $\delta N=0.2$ the $n-n$ correlation coefficient takes a non-zero value only at $N=N_{0}$ (which is possible only when $N_{0}$ is an even number). In this case interaction function $r(N)$ could be approximated by a Heaviside step function. We claim that this sharp transition from a lack of the fusion to complete fusion was considered rather to explore the mathematical limit behaviour of the model, than to produce some physical results.

\subsection{2 $n-n$ correlations}

The dependence of the $n-n$ correlation coefficient on the mean number of primary strings $\bar{N}$ is shown in Fig. 2 for $\delta N=20$, corresponding to slowly rising interaction function $r(N)$. The plot was obtained for different probability distributions $q(H)$ : binomial distributions with $\omega[N]=$ $\{0.1,0.5,1,1.5\}$ and Poisson distribution with $\omega[N]=2$. The results show a smooth transition from the no-fusion limit to the total-fusion limit with a single extremum point in this area - maximum for small $\omega[N]$ and minimum ${ }^{2}$ for large $\omega[N]$. From the previous experience [12] it was not expected that this transition could have a dip between two asymptotics. It leads us to conclude that, while both to the right and to the left of the dip (or hill) strings of a single type dominate, near the extremum point it is the interplay of the fusion and fluctuation effects. The general trend of the correlation growth with the rising fluctuations $\omega[N]$, which takes place in the model with independent emitters of one type [13], is well reproduced.

The analogous dependence of the $n-n$ correlation coefficient is shown in Fig. 3 for $\delta N=4$, which is associated with a rapidly growing with the number of strings probability of interaction. The drop of the correlations for large $\omega[N]$ is found to be more pronounced than in the previous

\footnotetext{
${ }^{2}$ The drop of the correlation coefficient in this area could be associated with a huge anti-correlation between $N_{1}$ and $N_{2}$.
} 
case. However, the main feature of this plot is a non-monotonic behaviour of the correlation coefficient for intermediate values of the fluctuation measure $\omega[N]=\{0.1 ; 0.5\}$, with three extremum points placed in the transition region. The presence of this wavy dependencies explains how the hill of correlations for $\omega[N]=0$ transforms to the dip for large $\omega[N]$.

Manifestation of these dependencies in an experimental data would be a clear signal of the string fusion phenomenon. One can connect model parameters $\bar{N}$ and $\omega[N]$ with some collision centrality estimators, because they change with varying of the class of centrality and its width. The common way to do it is to apply the Glauber model, calculate numbers of nucleon-participants and nucleon-spectators and parametrize $\bar{N}$ and $\omega[N]$ in terms of these numbers [20]. Leaving this approach for the future studies, we propose another method in this work. We calculate for each $\bar{N}$ average multiplicity $n_{\text {add }}$ in an additional window. It is supposed that this third window is placed far enough from two others to neglect correlations between observables in this window and others from one string.

In Fig. 4 and Fig. 5 the $n-n$ correlation coefficients are presented as functions of $n_{\text {add }}$ for the scope of parameters from Fig. 2 and 3 correspondingly. One can clearly see that for the $\delta N=20$ the behaviour of $b_{n-n}^{r e l}$ does not change after introducing of the new variable, while for the $\delta N=4$ strong oscillations take place in the extremum region. The presence of these oscillations makes it practically impossible to compare experimental data expressed in terms of $n_{\text {add }}$ with the model predictions.

Nevertheless, one can note, that the rise of centrality (growth of $\bar{N}$ or $n_{\text {add }}$ ) could lead as to the increase of the correlations (for small $\omega[N]$ ), as to the decrease (for large $\omega[N]$ ), however, the asymptotic value at large $\bar{N}\left(n_{\text {add }}\right)$ is always not greater than at small $\bar{N}\left(n_{\text {add }}\right)$.

\subsection{3 $p T-n$ correlations}

The results of the $p T-n$ correlation coefficient computation for $\overline{k_{1}}=0.3 \mathrm{GeV} / \mathrm{c}$ and $\delta \mathrm{N}=20$ are shown in Fig. 6. They demonstrate the transition from the negative values at small $\omega(N)$ to the positive ones at high $\omega(N)$. Similar behaviour has already been found both in experimental [21] and theoretical [22] studies. Moreover, $p T-n$ correlations tend to zero at small and high $\bar{N}$, representing their dependence exclusively on the fluctuations in the type of strings. It is this dependence that makes the long-range $p T-n$ correlations a unique tool to search for string fusion effects.

On the other hand, the non-monotonic behaviour found in the $n-n$ correlations for $\delta N=4$ (see Fig. 3) affects also the $p T-n$ correlations, as can be seen in Fig. 7. In this case several extremum points come into play in the transition area, giving possibility of obtaining negative values of the $p T-n$ correlation coefficient for large $\omega[N]$. Note also, that the dependence on the value of $\omega[N]$ is not linear at $N=N_{0}$.

In Fig. 8 and Fig. 9 the $p T-n$ correlation coefficient is presented as a function of $n_{\text {add }}$. Analogously to the $n-n$ case, the behaviour for $\delta N=20$ resemble that in Fig. 6. Only three curves are shown in Fig. 9 for better visibility due to the strong oscillations for $\omega[N]=0.1, \omega[N]=0.5$, $\omega[N]=1$. As in the $n-n$ case, direct comparison with experiment using the $n_{\text {add }}$ variable looks impossible. 


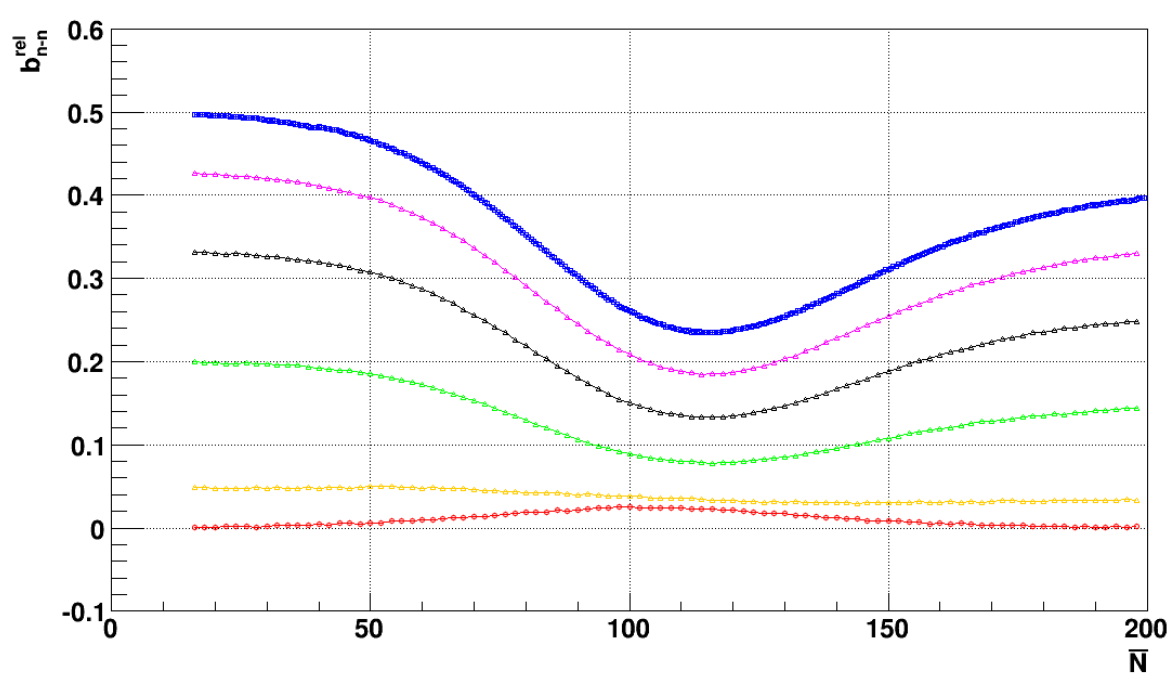

Figure 2: The $n-n$ correlation coefficient in the relative variables for $N_{0}=100, \bar{\mu}_{F}=\bar{\mu}_{B}=0.5, \delta N=20$ as a function of the mean number of primary strings $\bar{N}$. Red line corresponds to $\omega[N]=0$, yellow - $\omega[N]=0.1$, green $-\omega[N]=0.5$, black $-\omega[N]=1$, violet $-\omega[N]=1.5$, blue $-\omega[N]=2$. Statistical errors, calculated by the sub-sample method, for all points do not exceed 0.0005 units.

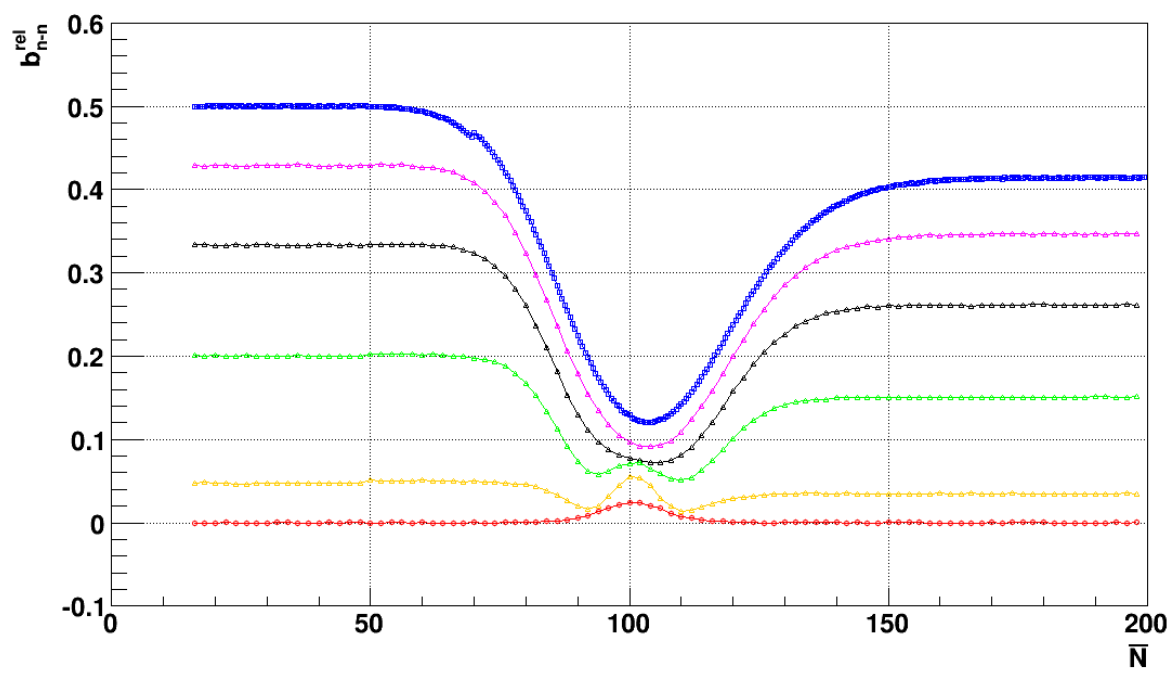

Figure 3: The $n-n$ correlation coefficient in the relative variables for $N_{0}=100, \bar{\mu}_{F}=\bar{\mu}_{B}=0.5, \delta N=4$ as a function of the mean number of primary strings $\bar{N}$. Red line corresponds to $\omega[N]=0$, yellow - $\omega[N]=0.1$, green $-\omega[N]=0.5$, black $-\omega[N]=1$, violet $-\omega[N]=1.5$, blue $-\omega[N]=2$. Statistical errors, calculated by the sub-sample method, for all points do not exceed 0.0005 units. 


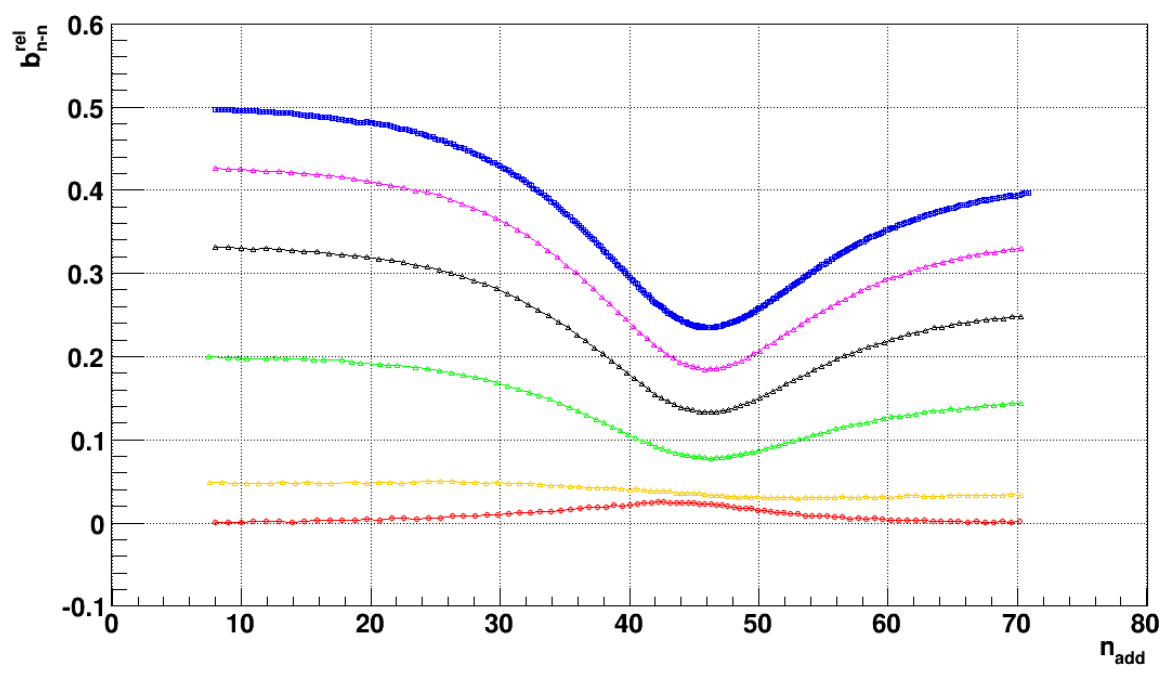

Figure 4: The $n-n$ correlation coefficient in the relative variables for $N_{0}=100, \bar{\mu}_{F}=\bar{\mu}_{B}=0.5, \delta N=20$ as a function of the average multiplicity in the additional window $n_{\text {add }}$. Red line corresponds to $\omega[N]=0$, yellow $-\omega[N]=0.1$, green $-\omega[N]=0.5$, black $-\omega[N]=1$, violet $-\omega[N]=1.5$, blue $-\omega[N]=2$. Statistical errors, calculated by the sub-sample method, for all points do not exceed 0.0005 units.

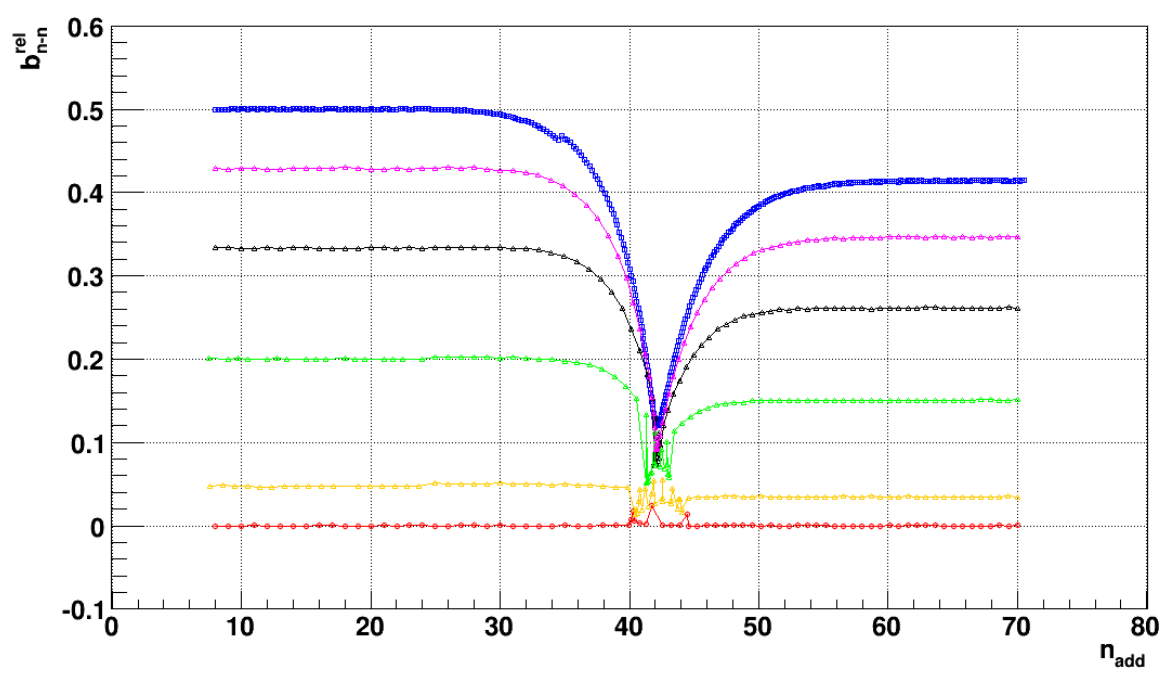

Figure 5: The $n-n$ correlation coefficient in the relative variables for $N_{0}=100, \bar{\mu}_{F}=\bar{\mu}_{B}=0.5, \delta N=4$ as a function of the average multiplicity in the additional window $n_{\text {add }}$. Red line corresponds to $\omega[N]=0$, yellow $-\omega[N]=0.1$, green $-\omega[N]=0.5$, black $-\omega[N]=1$, violet $-\omega[N]=1.5$, blue $-\omega[N]=2$. Statistical errors, calculated by the sub-sample method, for all points do not exceed 0.0005 units. 


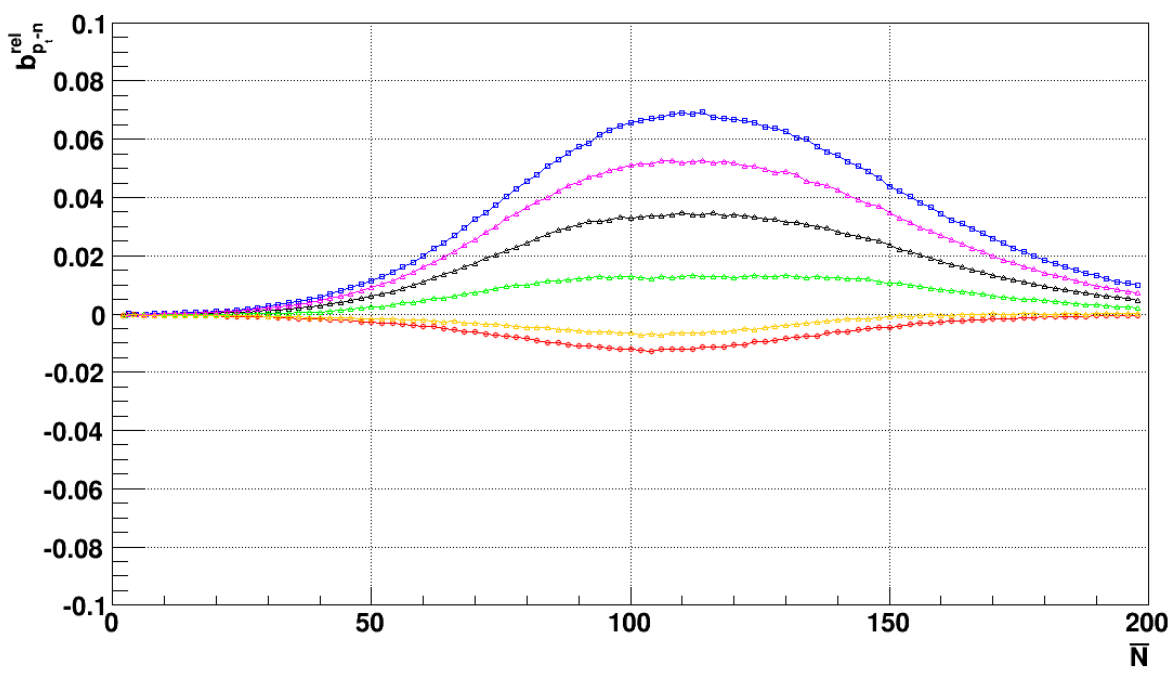

Figure 6: The $p T-n$ correlation coefficient in the relative variables for $N_{0}=100, \bar{\mu}_{F}=\bar{\mu}_{B}=0.5, \delta N=20$, $\overline{k_{1}}=0.3 \mathrm{GeV} / \mathrm{c}$ as a function of the mean number of primary strings $\bar{N}$. Red line corresponds to $\omega[N]=0$, yellow $-\omega[N]=0.1$, green $-\omega[N]=0.5$, black $-\omega[N]=1$, violet $-\omega[N]=1.5$, blue $-\omega[N]=2$. Statistical errors, calculated by the sub-sample method, for all points do not exceed 0.0002 units.

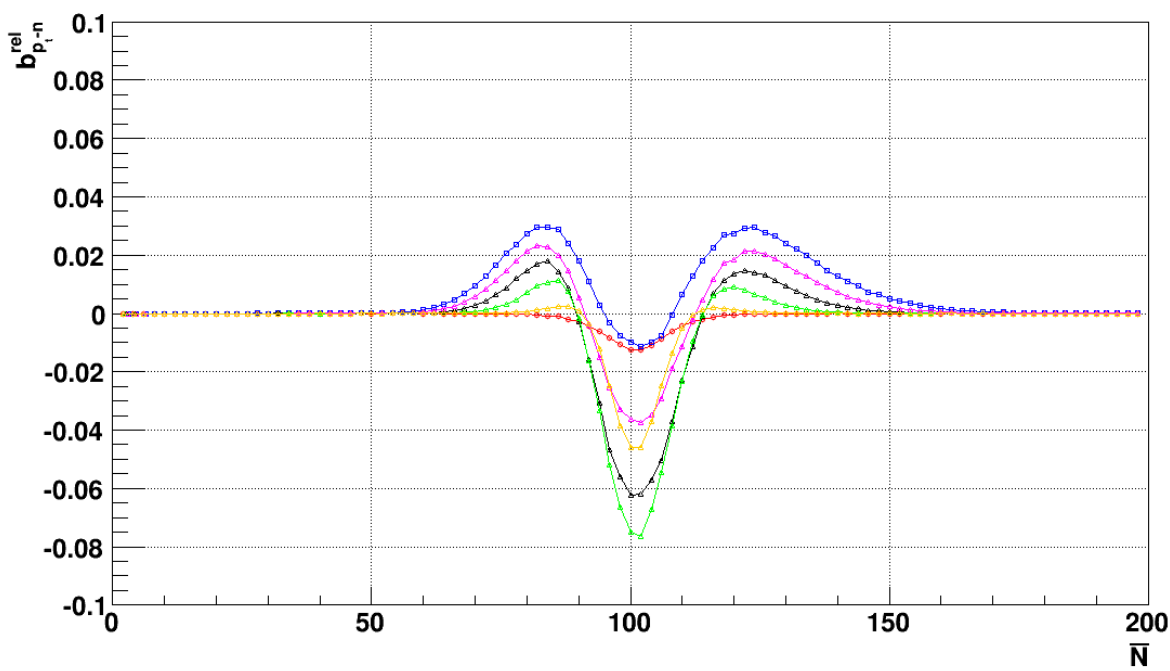

Figure 7: The $p T-n$ correlation coefficient in the relative variables for $N_{0}=100, \bar{\mu}_{F}=\bar{\mu}_{B}=0.5, \delta N=4$, $\overline{k_{1}}=0.3 \mathrm{GeV} / \mathrm{c}$ as a function of the mean number of primary strings $\bar{N}$. Red line corresponds to $\omega[N]=0$, yellow $-\omega[N]=0.1$, green $-\omega[N]=0.5$, black $-\omega[N]=1$, violet $-\omega[N]=1.5$, blue $-\omega[N]=2$. Statistical errors, calculated by the sub-sample method, for all points do not exceed 0.0002 units. 


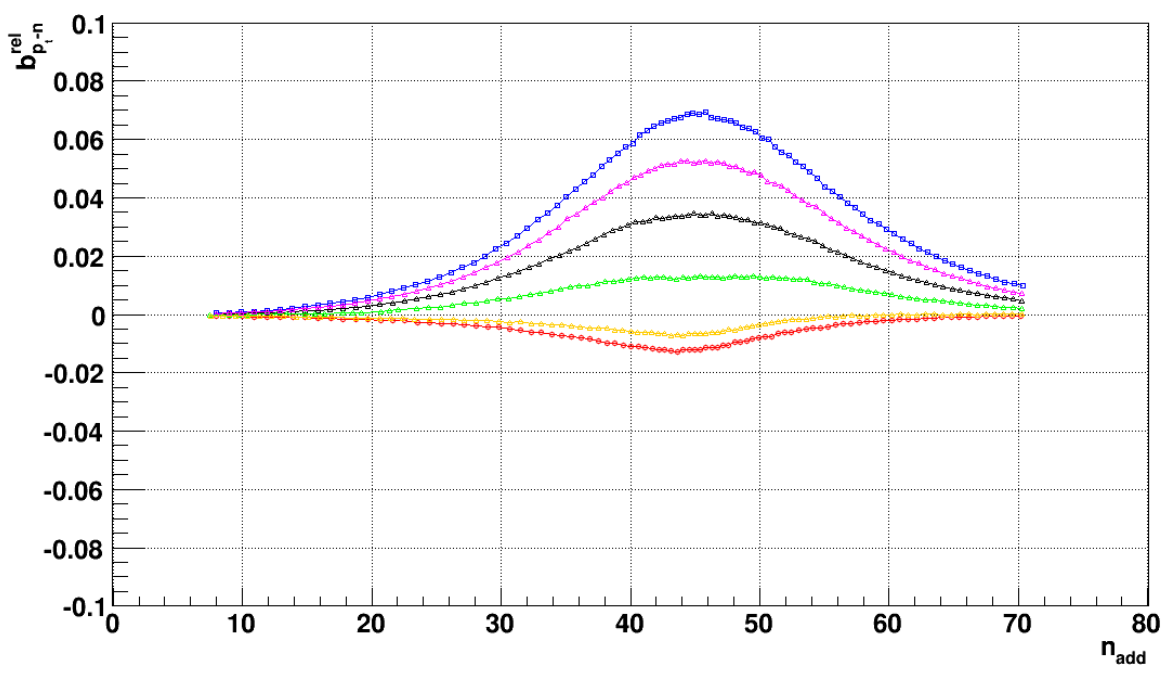

Figure 8: The $p T-n$ correlation coefficient in the relative variables for $N_{0}=100, \bar{\mu}_{F}=\bar{\mu}_{B}=0.5, \delta N=20$, $\overline{k_{1}}=0.3 \mathrm{GeV} / \mathrm{c}$ as a function of the average multiplicity in the additional window $n_{\text {add }}$. Red line corresponds to $\omega[N]=0$, yellow $-\omega[N]=0.1$, green $-\omega[N]=0.5$, black $-\omega[N]=1$, violet $-\omega[N]=1.5$, blue $\omega[N]=2$. Statistical errors, calculated by the sub-sample method, for all points do not exceed 0.0002 units.

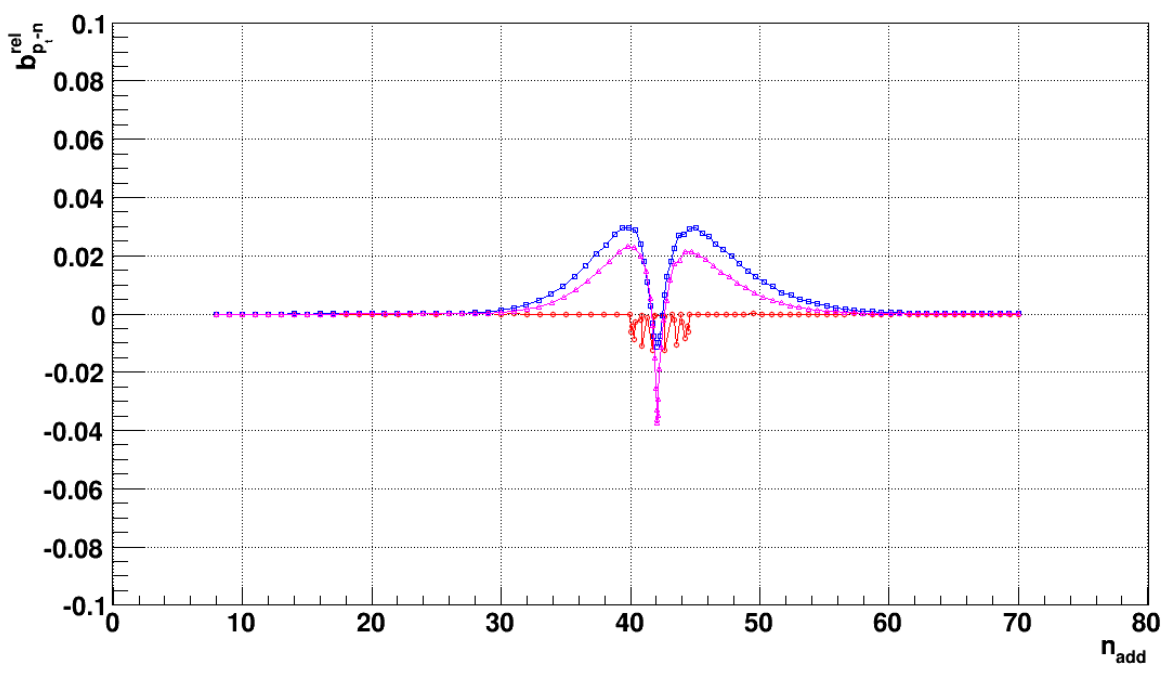

Figure 9: The $p T-n$ correlation coefficient in the relative variables for $N_{0}=100, \bar{\mu}_{F}=\bar{\mu}_{B}=0.5, \delta N=4$, $\overline{k_{1}}=0.3 \mathrm{GeV} / \mathrm{c}$ as a function of the average multiplicity in the additional window $n_{\text {add }}$. Violet line corresponds to $\omega[N]=0$, violet $-\omega[N]=1.5$, blue $-\omega[N]=2$. Statistical errors, calculated by the sub-sample method, for all points do not exceed 0.0002 units. 


\section{Summary and conclusions}

The Monte-Carlo realization of the model with strings of two types was developed and applied to the calculation of the $n-n$ and $p T-n$ correlation coefficients. Dependence of the interaction parameter $r$ on the number of primary strings $N$ was taken into account.

Simulation results in the case of the fixed number of primary strings $N$ showed a good agreement with the analytical consideration. Non-trivial behaviour of the $n-n$ correlation coefficient in the area where interaction between strings comes into play was obtained. The model enabled to describe also transition from the negative values of the $p T-n$ correlation coefficient to the positive ones. It turned out that both the $n-n$ and $p T-n$ correlations are very sensitive to the shape of the interaction function $r(N)$. Changing rate of growth of $r(N)$ from slow to rapid leads to appearance of additional extremum points.

For the more detailed comparison of the model predictions with the experimental results parameters of the simulations should be associated directly with information about collision centrality and multiplicity and transverse momentum spectra in rapidity windows. One of the possible centrality estimators, multiplicity in the additional rapidity window, was modelled. The obtained results for this parameter shows behaviour similar to the $\bar{N}$ dependence but with strong oscillations close to the extremum point. Connection of the model parameters with other centrality estimators like number of nucleon-participants is left for the future studies.

\section{References}

[1] A. Capella et al., Jets in small $p(T)$ hadronic collisions, universality of quark fragmentation, and rising rapidity plateaus, Phys.Lett. $\mathbf{B 8 1}$ (1979) 68.

[2] A. Capella et al., Dual parton model, Phys.Rept. 236 (1994) 225-329.

[3] A. B. Kaidalov, The quark-gluon structure of the pomeron and the rise of inclusive spectra at high energies, Phys.Lett. B116 (1982) 459.

[4] A. B. Kaidalov, and K. A. Ter-Martirosian, Pomeron as quark-gluon strings and multiple hadron production at SPS collider energies, Phys.Lett. B117 (1982) 247-251.

[5] K. Werner, Strings, pomerons and the VENUS model of hadronic interactions at ultrarelativistic energies, Phys. Rep. 232 (1993) 87.

[6] K. Werner et al., Gribov-Regge theory, partons, remnants, strings Ü and the EPOS model for hadronic interactions, Nucl. Phys. B Proc. suppl. 196 (2009) 36.

[7] M. A. Braun, and C. Pajares, Particle production in nuclear collisions and string interactions, Phys. Lett. B287 (1992) 154-158.

[8] M. A. Braun, and C. Pajares, A probabilistic model of interacting strings, Nucl. Phys. B390 (1993) $542-558$.

[9] N. S. Amelin et al., Long and short range correlations: a signature of string fusion, Phys. Rev. Lett. 73 (1994) 2813-2816.

[10] M. A. Braun et al., Correlations between multiplicities and average transverse momentum in the percolating color strings approach, Eur.Phys.J. C32 (2004) 535-546. 
[11] V. V. Vechernin, and R. S. Kolevatov, On multiplicity and transverse-momentum correlations in collisions of ultrarelativistic ions, Physics of Atomic Nuclei 70 (2007) 1797.

[12] E. Andronov, and V. Vechernin, The correlation between transverse momentum and multiplicity of charged particles in a two-component model, POS (QFTHEP 2013$) 054$ (2014).

[13] V. V. Vechernin, Long-Range Rapidity Correlations in the Model with Independent Emitters, in proceedings of The XX International Baldin Seminar on High Energy Physics Problems, Vol.2, JINR, Dubna, 2011, pp. 10-14, arXiv:1012.0214 [hep-ph].

[14] M. Derrick et al., Study of quark fragmentation in e+e-annihilation at 29-GeV: charged particle multiplicity and single particle rapidity distributions, Phys.Rev. D34 (1986) 3304.

[15] S. Uhlig et al., Observation of charged particle correlations between the forward and backward hemispheres in pp collisions at ISR energies, Nucl.Phys. $\mathbf{B 1 3 2}$ (1978) 15.

[16] V. N. Gribov, A reggeon diagram technique, Sov.Phys.JETP 26 (1968) 414-422.

[17] H. J. Drescher et al., Parton based Gribov-Regge theory, Phys.Rept. 350 (2001) 93-289.

[18] T. S. Biro et al., Color rope model for extreme relativistic heavy ion collisions, Nucl.Phys. B245 (1984) 449-468.

[19] A. Bialas, and W. Czyz, Conversion of color field into QQ matter in the central region of high-energy heavy ion collisions, Nucl.Phys. B267 (1986) 242.

[20] V.V. Vechernin, and H.S. Nguyen, Fluctuations of the number of participants and binary collisions in AA interactions at fixed centrality in the Glauber approach, Phys.Rev. $\mathbf{C 8 4}$ (2011) 054909.

[21] C. Alt et al. (NA49 Collaboration), G. A. Feofilov, R. S. Kondratiev, P. A. Naumenko, V. V. Vechernin, Long-Range Correlations in PbPb Collisions at $158 \mathrm{~A}^{*} \mathrm{GeV}$, in proceedings of The XVII International Baldin Seminar on High Energy Physics Problems, Vol. 1, JINR, Dubna, 2005, pp. 222-231.

[22] V. Kovalenko, and V. Vechernin, Long-range rapidity correlations in high energy AA collisions in Monte Carlo model with string fusion, EPJ Web Conf. 66 (2014) 04015. 